\title{
ALIMENTACIÓN Y MARGINACIÓN EN LA POBLACIÓN DEL NORESTE DE MÉXICO.
}

FEED AND MARGINATION IN THE POPULATION OF NORTHEAST MEXICO.

Galaviz Alarcón Silvia María*, Ramos Peña Esteban Gilberto*, Núñez Rocha Georgina Mayela*, Salas García Rogelio*.

* Facultad de Salud Pública y Nutrición, Universidad Autónoma de Nuevo León, México.

Citation: Galaviz Alarcón S.M., Ramos Peña E.G., Núñez Rocha G.M., Salas García R. (2019) Alimentación y marginación en población del noreste de México. Revista de Salud Pública y Nutrición, 18 (1), 9-14.

Editor: Esteban G. Ramos Peña, Dr. CS., Universidad Autónoma de Nuevo León, Facultad de Salud Pública y Nutrición, Monterrey Nuevo León, México. Copyright: (02019 Galaviz Alarcón S.M. et al. This is an open-access article distributed under the terms of Creative Commons Attribution License [CC BY 4.0], which permits unrestricted use, distribution, and reproduction in any medium, provided the original author and source are credited.

Competing interests: The authors have declared that no competing interests exist.

DOI: https://doi.org/10.29105/respyn18.1-2

Recibido: 02 de marzo 2019;

Aceptado: 05 de abril 2019

Email: esteban.ramosp@uanl.mx 


\title{
ALIMENTACIÓN Y MARGINACIÓN EN LA POBLACIÓN DEL NORESTE DE MÉXICO
}

\author{
Galaviz Alarcón Silvia María*, Ramos Peña Esteban Gilberto*, Núñez Rocha Georgina Mayela*, \\ Salas García Rogelio*. \\ * Universidad Autónoma de Nuevo León, Facultad de Salud Pública y Nutrición, México.
}

\section{RESUMEN}

Introducción: La alimentación puede ser afectada por varios factores, entre ellos la marginación que es multidimensional y expresa desigualdades. Objetivo: Determinar si la marginación está asociada a la calidad del patrón de consumo alimentario en población de 5-59 años en Nuevo León. Material y Métodos: Se utilizó la información de edad, municipio de residencia y 9 grupos de la frecuencia de consumo de alimentos (cereales, verduras, frutas, leche, carnes, leguminosas, embutidos, dulces y refrescos) de 5,195 individuos de 5-59 años participantes de la Encuesta Estatal de Salud y Nutrición de Nuevo León 2011/2012. La marginación se identificó por el lugar de residencia de cada participante y se clasificó de acuerdo con los grados de marginación propuestos por el CONAPO 2010, donde para el estado de Nuevo León se identificaron los grados alto, medio, bajo y muy bajo. La calidad del patrón de consumo alimentario se clasificó en 3 categorías: Inadecuada, Necesita Cambios y Adecuada. Resultados: Solo el $9.5 \%$ de la población presentó una calidad adecuada, mientras que el $84.5 \%$ necesita cambios. Más del $80 \%$ de la población de cada grado de marginación se ubicó en la categoría necesita cambios, en el grado de marginación alta el $14.3 \%$ de la población tiene calidad Inadecuada. Conclusiones: La calidad del patrón de consumo alimentario está asociado significativamente a la marginación.

Palabras Clave: Marginación, calidad de la alimentación, patrón alimentario, México.

\section{ABSTRACT}

Introduction: Feeding can be affected by several factors, including marginalization that is multidimensional and expresses inequalities. Objective: To determine if the marginalization is associated to the quality of the pattern of food consumption in the population of 5-59 years old in Nuevo León. Methods: We used the information of age, place of residence and 9 groups of the frequency of food consumption (cereals, vegetables, fruits, milk, meats, legumes, sausages, sweets and soft drinks), of 5,195 individuals of 5-59 years old participants of the State Health and Nutrition Survey of Nuevo León 2011/2012. The marginalization was identified by the place of residence of each participant and was classified according to the degrees of marginalization proposed by the CONAPO 2010, where for the state of Nuevo León were identified the grades high, medium, low and very low. The quality of the food consumption pattern was classified into 3 categories: Inadequate, Need Changes and Adequate. Results: Only $9.5 \%$ of the population presented an adequate quality, while $84.5 \%$ need changes. More than $80 \%$ of the population of each degree of marginalization was placed in the category needs changes, in the degree of high marginalization $14.3 \%$ of the population has inadequate quality. Conclusions: The quality of the pattern of food consumption is significantly associated with marginalization.

Key words: Marginalization, food quality, eating pattern, México. 


\section{Introducción}

La alimentación es afectada por diferentes factores biológicos, psicológicos, económicos y sociales. Las condiciones de la marginación fomentan una alimentación inadecuada y, por tanto, un estado de nutrición inadecuado, ya que, con situaciones como el bajo ingreso económico, el número elevado de integrantes en la familia, las malas condiciones de las viviendas, el pobre acceso a los alimentos, una educación alimentaria y nutricional escasa, mala higiene e inadecuadas prácticas de manipulación de alimentos, entre otras, afectan el consumo de alimentos (Ramos Peña, 2009).

La marginación es un fenómeno multidimensional que se expresa en desigualdad del progreso y excluye a grupos sociales de oportunidades de crecimiento y desarrollo. En el 2010 según el Consejo Nacional de Población (CONAPO), Nuevo León (NL) se ubicó en el índice de marginación muy bajo, sin embargo, cerca de 223 mil habitantes en el estado, principalmente en los municipios del sur, viven en comunidades con grados de marginación alta (CONAPO, 2015).

La epidemiología nutricional busca formas efectivas de evaluar la ingesta de alimentos a nivel poblacional, debido a que numerosos factores condicionan e influyen en la alimentación, su evaluación resulta difícil y compleja (Martínez, 2013; Serra, Aranceta, \& Mataix, 2006). Actualmente el interés se centra en la evaluación de patrones alimentarios y no en nutrientes, ya que, lo que se consume son combinaciones de alimentos y no nutrientes aislados (Hu, 2002; Martínez, 2013). Se han propuesto diferentes índices o métodos para evaluar la calidad de la dieta, algunos basados en nutrientes, otros en grupos de alimentos y algunos combinados (Gil, Martínez, \& Olza, 2015). La Calidad del Patrón de Consumo Alimentario (CPCA) es una adaptación del Índice de Alimentación Saludable (IAS) español, propuesto por NorteNavarro y Ortiz Moncada, 2011 (Norte \& Ortiz, 2011) que evalúa la calidad de la dieta de acuerdo a la concordancia del consumo de grupos de alimentos que recomienda la Sociedad Española de Nutrición Comunitaria (SENC) (Sociedad Española de Nutrición Comunitaria, 2015). La CPCA es un método rápido y fácil para conocer la calidad de la alimentación, ya que evalúa la frecuencia con la que los grupos de alimentos se consumen al contrastarlos con las recomendaciones establecidas. El objetivo de este estudio fue determinar si la marginación está asociada a la calidad del patrón de consumo alimentario en la población de 5-59 años en el estado de Nuevo León.

\section{Material y Métodos}

Estudio de diseño transversal, analítico, comparativo. Se utilizaron los datos de los participantes de la primera Encuesta Estatal de Salud y Nutrición 2011/2012 de Nuevo León (EESN-NL 2011/2012). En la encuesta se entrevistaron a 7,293 individuos, de los cuales solo se estudiaron a los participantes de 5-59 años $(5,211)$. Se eliminaron del presente análisis a 16 participantes con información incompleta en la frecuencia de consumo de alimentos o en alguna de las variables de interés, para este análisis se incluyeron a 5,195 individuos.

Para determinar el grado de marginación se utilizó el lugar de residencia de cada participante y se clasificó de acuerdo a los grados de marginación propuestos por el CONAPO 2010 (Consejo Nacional de Población (CONAPO), 2015), donde para el estado de Nuevo León se identificaron los grados alto, medio, bajo y muy bajo.

Para la creación de la CPCA se utilizaron 71 alimentos de la frecuencia de consumo de alimentos, conformados en 9 grupos (cereales, verduras, frutas, productos lácteos, carnes, leguminosas, embutidos, dulces y refrescos). Se asignó una puntuación a cada alimento de acuerdo con el cumplimiento de la recomendación de consumo semanal que establece la SENC 2015 en su Pirámide de la Alimentación Saludable 2015. Para los grupos de consumo diario (cereales, verduras, frutas y leche), la puntuación fue: (10 puntos si se consume 7 días, 7.5 de 5-6 días, 5 de 2-4 días, 2.51 día y 0 si nunca lo consume), para los grupos de consumo semanal (carnes y leguminosas) la puntuación fue: (10 puntos si se consume 2-4 días, 7.5 de 5-6 días, 5 solo 1 día, 2.5 los 7 días y 0 si nunca lo consume), para los grupos de consumo ocasional (embutidos, dulces y refrescos) la puntuación fue: (10 puntos si nunca consume, 7.5 solo 1 día, 5 de 24 días, 2.5 de 5-6 días y 0 si consume los 7 días). La categorización de la CPCA se estableció con base a la sumatoria de la puntuación ( $\geq 80$ Adecuada, 51-79 Necesita cambios y $\leq 50$ Inadecuada) (Norte \& Ortiz, 2011). 
El análisis estadístico se llevó a cabo por medio de descriptivos y una prueba de chi cuadrada para determinar la asociación de las variables. La significancia estadística se estableció con un valor $p$ $<0.05$ y se utilizó el paquete estadístico NCSS 10. Las diferencias entre cada grupo de las estratificaciones de detectaron a través de los intervalos con el $95 \%$ de confianza.

\section{Resultados}

El $58.5 \%$ de la población eran mujeres y el $41.5 \%$ hombres. La media de edad de la población fue de $28.3 \pm 16.3$ años (DS). El $61.1 \%$ se ubicaron en el estrato de muy baja marginación, $13.0 \%$ baja, $25.4 \%$ media y $0.5 \%$ alta. En cuanto a la calidad del patrón de consumo alimentario, el $9.5 \%$ tuvo CPCA adecuada, el $6.0 \%$ inadecuada y el $84.5 \%$ necesita cambios.

Al estratificar la CPCA por grupos de edad, se observó que en los tres grupos los porcentajes más altos se ubican en necesidad de cambios en la alimentación, siendo más alta la proporción en el grupo de 5-9 años (87.8\%) (Ver tabla 1).

Se encontró que la CPCA está asociada al sexo $(\mathrm{p}<0.05)$, el porcentaje de mujeres con CPCA adecuada es mayor que los hombres. Al estratificar la población por grupos de edad, también se encuentra asociada la CPCA a los grupos de edad $(p<0.05)$, es mayor el porcentaje de individuos del grupo de 10 a 19 años con CPCA inadecuada que los del grupo de 5 a 9 años, mientras que en la CPCA adecuada es mayor el porcentaje de los de 20 a 59 años que los del grupo de 10 a 19 años (Ver tabla 1).

En cuanto a los grupos de alimentos, los porcentajes más altos que se ubicaron en la CPCA inadecuada fueron los grupos de refrescos, frutas, productos lácteos y leguminosas, los porcentajes más altos en CPCA adecuada fue en los grupos de cereales, carnes y dulces (Ver tabla 1).
Tabla 1. Calidad del Patrón de Consumo Alimentario según población, sexo, edad y grupo de alimentos en hogares de Nuevo León

\begin{tabular}{|c|c|c|c|c|c|c|}
\hline & \multicolumn{2}{|c|}{ Inadecuada } & \multicolumn{2}{|c|}{ Necesita Cambios } & \multicolumn{2}{|c|}{ Adecuada } \\
\hline & $\%$ & $\mathrm{IC}_{95}$ & $\%$ & $\mathrm{I}_{95}$ & $\%$ & $\mathrm{IC}_{95}$ \\
\hline General* & 6.0 & $5.0-7.0$ & 84.5 & $83.5-85.5$ & 9.5 & $8.5-10.5$ \\
\hline \multicolumn{7}{|l|}{ Sexo+ } \\
\hline Hombres $(\mathrm{n}=2155)$ & 7.1 & $6.0-8.2$ & 84.4 & $82.4-86.4$ & 8.5 & $7.3-9.7$ \\
\hline Mujeres ( $n=3040$ ) & 5.2 & $4.2-6.1$ & 84.5 & $83.5-85.5$ & 10.2 & $8.9-11.5$ \\
\hline \multicolumn{7}{|l|}{ Grupo de Edad+ } \\
\hline $5-9 \quad(n=763)$ & 5.2 & $3.2-7.2$ & 86.5 & $84.5-88.5$ & 8.3 & $6.3-10.3$ \\
\hline $10-19(n=1319)$ & 8.3 & $7.3-9.3$ & 84.3 & $82.3-86.3$ & 7.4 & $6.4-8.4$ \\
\hline $20-59 \quad(n=3113)$ & 5.2 & $4.2-6.2$ & 84.1 & $83.3-85.3$ & 10.8 & $9.8-11.8$ \\
\hline \multicolumn{7}{|l|}{ Gpo. De Alimentos* } \\
\hline Cereales & 2.5 & $25-2.5$ & 12.6 & 11.6 - 13.6 & 85.0 & $84.0-86.0$ \\
\hline Verduras & 16.9 & $15.9-17.9$ & 29.0 & $28.0-30.0$ & 54.0 & $53.0-55.0$ \\
\hline Frutas & 39.9 & $38.9-40.9$ & 50.4 & $49.4-51.4$ & 9.7 & $8.7-10.7$ \\
\hline Productos Lácteos & 23.5 & $22.5-24.5$ & 34.8 & $33.8-35.8$ & 41.7 & $40.7-427$ \\
\hline Carnes & 9.1 & $8.3-9.9$ & 40.1 & $2.1-22.3$ & 50.8 & $35.3-66.3$ \\
\hline Leguminosas & 59.1 & $57.8-60.4$ & 19.4 & $7.2-31.6$ & 21.5 & $8.8-34.2$ \\
\hline Embutidos & 12.2 & $11.3-13.1$ & 59.3 & $44.1-74.5$ & 28.5 & $14.5-42.5$ \\
\hline Dulces & 4.8 & $3.8-5.8$ & 28.3 & $27.3-29.3$ & 66.9 & $65.9-67.9$ \\
\hline Refrescos & 61.7 & $60.7-62.7$ & 18.8 & $17.8-19.8$ & 19.4 & $18.4-20.4$ \\
\hline
\end{tabular}

Al contrastar la CPCA con los grados de marginación, se encontró que se encuentran asociados $(p<0.05)$. En las categorías de CPCA adecuada y necesita cambios no se encuentran diferencias en el porcentaje de población de cada nivel de marginación, mientras que en la categoría de CPCA adecuada, los porcentajes difieren entre las categorías de las marginaciones media, baja y muy baja (ver tabla 2)

Tabla 2. Población por Calidad del Patrón de Consumo Alimentario, según marginación

\begin{tabular}{|c|c|c|c|c|c|c|}
\hline \multirow{3}{*}{ Marginación } & \multicolumn{6}{|c|}{ CPCA } \\
\hline & \multicolumn{2}{|c|}{ Inadecuada } & \multicolumn{2}{|c|}{ Necesita Cambios } & \multicolumn{2}{|c|}{ Adecuada } \\
\hline & $\%$ & $\mathrm{IC}_{95}$ & $\%$ & $\mathrm{IC}_{95}$ & $\%$ & $\mathrm{IC}_{95}$ \\
\hline Alta $(n=28)$ & 14.3 & $1.4-27.2$ & 82.1 & $68.0-96.2$ & 3.6 & $0-10.5$ \\
\hline Media $(n=1321)$ & 7.2 & $5.9-8.5$ & 88.0 & $86.3-89.7$ & 4.8 & $3.7-5.9$ \\
\hline Baja $(n=673)$ & 5.8 & $4.1-7.5$ & 85.9 & $83.3-88.5$ & 8.3 & $6.3-10.3$ \\
\hline Muy Baja $(n=3173)$ & 5.4 & $4.6-6.1$ & 82.7 & $81.4-84.0$ & 11.8 & $10.7-12.9$ \\
\hline
\end{tabular}

Cuando se realiza la contrastación entre CPCA y el sexo de la población estratificada por niveles de marginación, se encontró asociación entre las dos variables $(\mathrm{p}<0.05)$. En las categorías de CPCA inadecuada y necesitan cambios no se encuentras diferencias en los porcentajes entre los niveles de marginación, mientras que en la categoría de CPCA adecuada, la diferencia está entre los niveles medio y muy baja de marginación (ver tabla 3 ) 
Tabla 3. Población por Calidad del Patrón de Consumo Alimentario, por sexo según marginación

\begin{tabular}{|c|c|c|c|c|c|c|}
\hline \multirow{2}{*}{$\begin{array}{c}\text { Marginación por } \\
\text { sexo }\end{array}$} & \multicolumn{2}{|c|}{ Inadecuada } & \multicolumn{2}{|c|}{ Necesita Cambios } & \multicolumn{2}{|c|}{ Adecuada } \\
\hline & $\%$ & $\mathrm{IC}_{95}$ & $\%$ & $\mathrm{IC}_{95}$ & $\%$ & $\mathrm{IC}_{95}$ \\
\hline \multicolumn{7}{|l|}{ Hombres * } \\
\hline Alta $(n=12)$ & 25.0 & $0-50$ & 75.0 & $50-100$ & 0.0 & 0.0 \\
\hline Media $(n=596)$ & 7.9 & $5.9-9.9$ & 88.3 & $85.3-91.3$ & 3.9 & $3.5-4.3$ \\
\hline Baja $(n=263)$ & 6.5 & $3.5-9.5$ & 85.9 & $81.9-89.9$ & 7.6 & $4.6-10.6$ \\
\hline Muy Baja $(n=1284)$ & 6.6 & $6.3-6.9$ & 82.4 & $80.4-84.4$ & 11.0 & $9.0-13.0$ \\
\hline \multicolumn{7}{|l|}{ Mujeres $^{\star}$} \\
\hline Alta $(n=16)$ & 6.3 & $0-18.3$ & 87.5 & $71.5-100$ & 6.3 & $0-18.3$ \\
\hline Media $(n=725)$ & 6.6 & $4.6-8.6$ & 87.9 & $85.9-89.9$ & 5.5 & $3.5-7.5$ \\
\hline Baja $(n=410)$ & 5.4 & $3.4-7.4$ & 85.9 & $82.9-88.9$ & 8.8 & $5.8-11.8$ \\
\hline Muy Baja $(n=1889)$ & 4.6 & $3.6-5.6$ & 83.0 & $81.0-85.0$ & 12.4 & $11.4-13.4$ \\
\hline
\end{tabular}

Al contrastar la CPCA y el grupo de edad de la población estratificado por niveles de marginación, se encontró asociación entre las dos variables solo en el grupo de 20 a 59 años ( $<<.05)$. Las diferencias de porcentajes se encuentran en la categoría de CPCA adecuada, en donde, el porcentaje es mayor en el estrato de marginación muy baja respecto a la baja y la media (ver tabla 4).

Tabla 4. Población por Calidad del Patrón de Consumo Alimentario, según marginación por grupo de edad

\begin{tabular}{|c|c|c|c|c|c|c|}
\hline \multirow{3}{*}{$\begin{array}{l}\text { Marginación por } \\
\text { grupo de edad }\end{array}$} & \multicolumn{6}{|c|}{ Calidad del Patrón de Consumo Alimentario } \\
\hline & \multicolumn{2}{|c|}{ Inadecuada } & \multicolumn{2}{|c|}{ Necesita Cambios } & \multicolumn{2}{|c|}{ Adecuada } \\
\hline & $\%$ & $\mathrm{IC}_{95}$ & $\%$ & $\mathrm{IC}_{95}$ & $\%$ & $\mathrm{IC}_{95}$ \\
\hline \multicolumn{7}{|l|}{$5-9$} \\
\hline Alta $(n=2)$ & 0.0 & 0.0 & 100.0 & 100.0 & 0.0 & 0.0 \\
\hline Media $(n=248)$ & 8.5 & $5.3-11.9$ & 85.5 & $81.1-89.9$ & 6.0 & $3.0-8.9$ \\
\hline Baja $(n=87)$ & 4.6 & $0.2-9.0$ & 86.2 & $78.9-93.4$ & 9.2 & $3.1-15.3$ \\
\hline Muy Baja $(n=426)$ & 3.5 & $1.5-5.2$ & 87.1 & $83.9-90.2$ & 9.4 & $6.6-12.7$ \\
\hline \multicolumn{7}{|l|}{$10-19$} \\
\hline Alta $(n=7)$ & 28.6 & $0.0-62.8$ & 71.4 & $37.9-100$ & 0.0 & $0.0-0.0$ \\
\hline Media $(n=290)$ & 6.9 & $4.0-9.8$ & 88.6 & $84.9-92.3$ & 4.5 & $2.1-6.9$ \\
\hline Baja $(n=175)$ & 8.6 & $4.4-12.7$ & 82.3 & $76.6-87.9$ & 9.1 & $4.8-13.4$ \\
\hline Muy Baja $(n=847)$ & 8.5 & $6.6-10.4$ & 83.4 & $80.9 \cdot 85.9$ & 8.1 & $6.3-9.9$ \\
\hline \multicolumn{7}{|l|}{$20-59^{*}$} \\
\hline Alta $(n=19)$ & 10.5 & $0.0-24.3$ & 84.2 & $67.9-100$ & 5.3 & $0.0-15.4$ \\
\hline Media $(n=783)$ & 6.9 & $5.1-8.7$ & 88.6 & $86.4-90.8$ & 4.5 & $3.1-5.9$ \\
\hline Baja $(n=411)$ & 4.9 & $2.8-7.0$ & 87.3 & $84.1-90.5$ & 7.8 & $5.2-10.4$ \\
\hline Muy Baja $(n=1900)$ & 4.5 & $3.9-5.9$ & 81.5 & $79.8-83.2$ & 14.1 & $12.5-15.7$ \\
\hline
\end{tabular}

${ }^{*} \mathrm{Ch}^{2} \mathrm{p}<0.05$

Se encontró que la CPCA está asociada a la marginación en todos los grupos de alimentos $(\mathrm{p}<0.05)$. El grupo de cereales presenta los porcentajes más altos de población con CPCA adecuada, mientras que en, las frutas, lácteos, leguminosas y refrescos lo tienen en la CPCA inadecuada en algunos niveles de marginación. En los grupos de alimentos de verduras, frutas, productos lácteos y carnes, el porcentaje de población con CPCA adecuada es más alto en el nivel de marginación muy baja respecto al nivel alto de marginación (ver tabla 5)

\begin{tabular}{|c|c|c|c|c|c|c|}
\hline \multirow{2}{*}{$\begin{array}{l}\text { Marginación por grupo } \\
\text { de alimento }\end{array}$} & \multicolumn{2}{|c|}{ Inadecuada } & \multicolumn{2}{|c|}{ Nec esita Cambios } & \multicolumn{2}{|c|}{ Adecuada } \\
\hline & $\%$ & $\mathrm{IC}_{95}$ & $\%$ & $\mathrm{IC}_{95}$ & $\%$ & $\mathrm{IC}_{95}$ \\
\hline \multicolumn{7}{|l|}{ Cereales ${ }^{*}$} \\
\hline Alta $(n=28)$ & 3.6 & $1.8-5.4$ & 0.0 & 0.0 & 96.4 & $89.4-100$ \\
\hline Media $(n=1321)$ & 2.4 & $1.4-3.4$ & 7.9 & $6.9-8.9$ & 89.6 & $87.6-91.6$ \\
\hline Baja $(n=673)$ & 1.9 & $0.9-2.9$ & 9.4 & $7.4-11.4$ & 88.7 & $86.7-90.7$ \\
\hline Muy Baja $(n=3173)$ & 2.6 & $1.6-3.6$ & 15.3 & $14.3-16.3$ & 82.2 & $81.2-83.2$ \\
\hline \multicolumn{7}{|l|}{ Verduras* } \\
\hline Alta $(n=28)$ & 32.1 & $15.1-49.1$ & 39.3 & $21.3-57.3$ & 28.6 & $11.6-45.6$ \\
\hline Media $(n=1321)$ & 17.9 & $15.9-19.9$ & 26.0 & $24.0-28.0$ & 56.2 & $53.0-59.0$ \\
\hline Baja $(n=673)$ & 13.4 & $10.4-16.4$ & 29.1 & $26.1-32.1$ & 57.5 & $53.5-61.5$ \\
\hline Muy Baja $(n=3173)$ & 17.2 & $16.2-18.2$ & 30.2 & $28.2--32.2$ & 52.6 & $50.6-54.6$ \\
\hline \multicolumn{7}{|l|}{ Frutas* } \\
\hline Alta $(n=28)$ & 60.7 & $42.7-78.7$ & 35.7 & $17.7-53.7$ & 3.6 & $1.8-5.4$ \\
\hline Media $(n=1321)$ & 51.1 & $48.0-54.1$ & 43.8 & $40.8-46.8$ & 5.1 & $4.1-6.1$ \\
\hline Baja $(n=673)$ & 42.2 & $38.2-46.2$ & 48.6 & $44.6-52.6$ & 9.2 & $7.2-11.2$ \\
\hline Muy Baja $(n=3173)$ & 34.5 & $32.5-36.5$ & 53.7 & $51.7-55.7$ & 11.8 & $10.8-12.8$ \\
\hline \multicolumn{7}{|l|}{ Productos Lácteos* } \\
\hline Alta $(n=28)$ & 60.7 & $42.7-78.7$ & 25.0 & $9.0-41.0$ & 14.3 & $1.0-27.3$ \\
\hline Media $(n=1321)$ & 37.0 & $34.0-40.0$ & 32.7 & $29.7-35.7$ & 30.3 & $28.3-32.3$ \\
\hline Baja $(n=673)$ & 23.0 & $20.0-26.0$ & 34.6 & $30.6-38.6$ & 42.3 & $38.3-46.3$ \\
\hline Muy Baja $(n=3173)$ & 17.7 & $16.7-18.7$ & 35.8 & $33.8-37.8$ & 46.5 & $44.5-48.5$ \\
\hline \multicolumn{7}{|l|}{ Carnes* $^{*}$} \\
\hline Alta $(n=28)$ & 14.3 & $1.3-27.8$ & 67.9 & $50.6-85.2$ & 17.9 & $15.9-19.9$ \\
\hline Media $(n=1321)$ & 16.0 & $14.0-18.0$ & 57.4 & $54.7-60.1$ & 26.6 & $24.2-29.0$ \\
\hline Baja $(n=673)$ & 8.2 & $4.5-11.9$ & 37.4 & $33.7-41.1$ & 54.4 & $50.6-58.2$ \\
\hline Muy Baja $(n=3173)$ & 6.4 & $5.6-7.3$ & 33.2 & $31.6-34.8$ & 60.4 & $58.7-62.1$ \\
\hline \multicolumn{7}{|l|}{ Leguminosas* } \\
\hline Alta $(n=28)$ & 67.9 & $50.6-85.2$ & 10.7 & $0-22.2$ & 21.4 & $6.2-36.6$ \\
\hline Media $(n=1321)$ & 72.2 & $69.8-74.6$ & 17.3 & $15.3-19.3$ & 10.5 & $8.9-12.2$ \\
\hline Baja $(n=673)$ & 66.1 & $62.5-69.7$ & 15.2 & $12.5-17.9$ & 18.7 & $15.8-21.7$ \\
\hline Muy Baja $(n=3173)$ & 52.1 & $50.4-53.8$ & 21.3 & $19.9-22.7$ & 26.6 & $25.1-28.1$ \\
\hline \multicolumn{7}{|l|}{ Embutidos* } \\
\hline Alta $(n=28)$ & 0.0 & $0-0$ & 17.9 & $15.9-19.9$ & 82.1 & $67.9-96.3$ \\
\hline Media $(n=1321)$ & 8.6 & $7.1-10.1$ & 33.7 & $31.2-36.2$ & 57.7 & $55.0-60.4$ \\
\hline Baja $(n=673)$ & 11.4 & $9.0-13.8$ & 64.6 & $61.0-68.2$ & 23.9 & $20.7-27.1$ \\
\hline Muy Baja $(n=3173)$ & 13.9 & $12.7-15.1$ & 69.2 & $67.6-70.8$ & 16.9 & $15.6-18.2$ \\
\hline \multicolumn{7}{|l|}{ Dulces $^{*}$} \\
\hline Alta $(n=28)$ & 0.0 & 0.0 & 14.3 & $1.3-27.3$ & 85.7 & $72.7-98.7$ \\
\hline Media $(n=1321)$ & 3.2 & $2.2-4.2$ & 23.4 & $21.4-25.4$ & 73.4 & $71.4-75.4$ \\
\hline Baja $(n=673)$ & 4.5 & $2.5-6.5$ & 27.5 & $24.5-30.5$ & 68.1 & $64.1-72.1$ \\
\hline Muy Baja $(n=3173)$ & 5.6 & $4.6-6.6$ & 30.6 & $28.6-32.6$ & 63.8 & $61.8-65.8$ \\
\hline \multicolumn{7}{|l|}{ Refrescos ${ }^{*}$} \\
\hline Alta $(n=28)$ & 64.3 & $46.3-82.3$ & 28.6 & $11.6-45.6$ & 7.1 & $0-17.1$ \\
\hline Media $(n=1321)$ & 61.9 & $58.9-64.9$ & 16.2 & $14.2-18.2$ & 21.9 & $19.9-23.9$ \\
\hline Baja $(n=673)$ & 67.9 & $63.9-71.9$ & 15.3 & $12.3-18.3$ & 16.8 & $13.8-19.8$ \\
\hline Muy Baja $(n=3173)$ & 60.3 & $58.3-62.3$ & 20.6 & $19.6-21.6$ & 19.1 & $18.1-20.1$ \\
\hline
\end{tabular}

Fuente: EESN-NL 2011/2012

${ }^{*} \mathrm{Chi}^{2} \mathrm{p}<.05$

\section{Discusión}

Hemos encontrado que la CPCA está asociada a los diferentes grados de marginación. La alimentación en general es afectada por diferentes determinantes, que condicionan la calidad de los alimentos que se consumen; estos determinantes, cuya combinación dan forma a las categorías de la marginación, tendrían mayor o menor influencia en el acceso a los alimentos dependiendo del grado de marginación en que se encuentren las comunidades. 
El estudio en población española (Norte y Ortiz, 2011) mostró que el $72 \%$ del total de la muestra necesitaba cambios en su alimentación y que la alimentación de las clases sociales más bajas era menos saludable. Nuestros resultados refieren mayor porcentaje (84.5\%, IC95 83.5-85.5) de población que requiere cambio en su alimentación y que solo (3.6\%) de la población del estrato de marginación alto tiene CPCA adecuada.

Otro estudio encontró que la población con grado de marginación alto tenía menor variedad de alimentos en su dieta, en comparación con los sujetos del grado muy bajo (208 alimentos vs. 404) (Ramos-Peña et al., 2007), esto puede deberse a diferentes factores que influyen en todo el proceso alimentario, desde la capacidad económica, la oferta y disposición de alimentos en las regiones, la calidad e inocuidad de los alimentos, la cultura y hábitos de quien compra y prepara los alimentos entre otros.

El estudio en población del Estado de Veracruz, encontró que en ninguno de los niveles socioeconómicos los alimentos básicos requeridos para una buena alimentación superaron el $50 \%$ y el $80 \%$ de la población ha recibido recomendaciones médicas de hacer cambios en su alimentación (Del Ángel \& Villagómez, 2013), lo que concuerda con los datos de nuestra investigación que el $84.5 \%$ necesita realizar cambios. Recientemente un estudio realizado en población adulta mexicana donde evaluaron la calidad de la dieta por medio de una aproximación cuantitativa al cumplimiento de los diferentes componentes de una alimentación saludable (suficiente, balanceada, completa, variada e inocua), mencionados en la Norma Oficial Mexicana 043, de acuerdo a distintas fuentes de recomendaciones de nutrientes y grupos de alimentos, encontró que la puntuación media de dicho índice fue de 68.5 \pm 9.3 , (Macedo, Márquez, Fernández, \& Vizmanos, 2016) lo cual es similar a la media encontrada en este estudio $(66.6 \pm 10.1)$, sin embargo, no muestran clasificación de categorías de la CPCA.

Otros estudios en Colombia han reportado que, principalmente las familias que viven en situación de pobreza se encuentran en inseguridad alimentaria (Aguirre-Acevedo \& Álvarez, 2012; Prada, Herrén, \& Ortíz, 2008). En un estudio realizado en el municipio de Girón Santander, evaluaron a familias en situación de pobreza y desplazamiento, registrando que el $95 \%$ de las familias se encontraban en inseguridad alimentaria y el $34 \%$ manifestaban no tener acceso a la compra de alimentos (Prada et al., 2008). Así mismo, Álvarez y Aguirre 2012, clasificaron a las familias en conglomerados de acuerdo a su nivel de pobreza y reportaron que el $18 \%$ de las familias se encontraban en el conglomerado 4, (de mayor pobreza), y estas mismas familias presentaban mayores prevalencias de inseguridad alimentaria leve o moderada (AguirreAcevedo \& Álvarez, 2012).

Hemos encontrado que en el grupo de adolescentes los grados de marginación alta y media presentan CPCA inadecuada, este resultado es consistente con el estudio de Béghin, 2014, el cual analizó datos de 1,768 adolescentes de ocho diferentes países europeos participantes del estudio Healthy Lifestyle in Europe by Nutrition in Adolescence (HELENA study) donde encontraron que, aquellos adolescentes con padres de menor nivel socioeconómico mostraron menores puntuaciones en la calidad de la dieta (Béghin et al., 2014). Así mismo, Darmon, 2008 menciona que, en los Estados Unidos, los niños $\mathrm{y}$ adolescentes de hogares de bajo nivel socioeconómico consumen menos frutas y verduras y una variedad más limitada de productos, lo que representa una dieta inadecuada (Darmon \& Drewnowski, 2008).

La inadecuada calidad de la dieta en poblaciones en comunidades con diferentes grados de marginación puede explicarse debido a que los factores que influyen en la selección de los alimentos de las personas son muy variados, complejos y multifactoriales (Darmon \& Drewnowski, 2008). En este sentido la Secretaría de Desarrollo Social (SEDESOL) en México en su documento diagnóstico y propuesta de atención del programa de comedores comunitarios en 2014, afirma que las condiciones de marginación guardan estrecha relación con la pobreza extrema de alimentación y menciona que los tres factores que más influyen son los relacionados a la disponibilidad y acceso a los alimentos, los bajos o insuficientes ingresos económicos y el consumo inadecuado y bajo aprovechamiento de nutrientes (SEDESOL, 2014).

Nuestro estudio presenta algunas fortalezas, principalmente el tamaño de la muestra, que incluyó 3 grupos de edades diferentes, así como la fácil 
utilización de datos secundarios, esencialmente los provenientes de la frecuencia de consumo de alimentos, sin embargo, como limitaciones, tenemos que, la frecuencia de consumo de alimentos no era semicuantitativa, y la guía de alimentación utilizada para evaluar la calidad de la dieta no era específica para población mexicana.

\section{Conclusiones}

En conclusión, la CPCA está asociada a la marginación. Es importante conocer la situación alimentaria de los más vulnerables, para contribuir a la generación y ejecución de nuevas estrategias que garanticen que, los alimentos ricos en nutrientes y una dieta de calidad estén igualmente disponibles para todos. Se necesitan más estudios que evalúen la calidad de la dieta de las personas en marginación, por medio de diferentes procedimientos, que sean comparados y que esta información inspire la generación de una ley alimentaria que proteja, respalde y haga realidad el derecho a la alimentación de todos los seres humanos.

\section{Bibliografía}

Aguirre-Acevedo, D. C., \& Alvarez, M. C. (2012). Perfil de la pobreza en hogares que participan en un programa de complementación alimentaria. Revista de Salud Pública, 14(3), 365-376. Béghin, L., Dauchet, L., De Vriendt, T., Cuenca-García, M., Manios, Y., Toti, E., ... Dallongeville, J. (2014). Influence of parental socio-economic status on diet quality of European adolescents: results from the HELENA study. British Journal of Nutrition, 111(7), 1303-1312.

CONAPO. (2010). Índice de Marginación por entidad federativa y municipio 2010. Recuperado el 18 de semptiembre de 2015 de: http://www.conapo.gob.mx/en/CONAPO/Indices_de_ Marginacion_2010_por_entidad_federativa_y_munici pio

Darmon, N., \& Drewnowski, A. (2008). Does social class predict diet quality? The American Journal of Clinical Nutrition, 87(5), 1107-17. https://doi.org/87/5/1107 [pii]

Del Ángel, A. L., \& Villagómez, J. A. (2013). Alimentación, salud y pobreza en áreas marginadas urbanas: caso Veracruz-Boca del Río , Veracruz , México. Estudios Sociales, 22(44), 11-35.

Gil, Á., Martínez, E., \& Olza, J. (2015). Indicators for the evaluation of the diet quality. Nutricion Hospitalaria, 31(3), 128-144.
Hu, F. B. (2002). Dietary pattern analysis: a new direction in nutritional epidemiology. Current Opinion in Lipidology, 13 (1), 3-9.

Macedo, G., Márquez, F., Fernández, J., \& Vizmanos, B. (2016). The reproducibility and relative validity of a Mexican diet quality index (ICDMx) for the assessment of the habitual diet of adults. Nutrients, 8 (9), 1-18.

Martínez, M. Á. (2013). Conceptos de Salud Pública y Estrategias Preventivas. Un manual para ciencias de la salud. Barcelona, España: Elsevier.

Norte, A. I., \& Ortiz, R. (2011). Calidad de la dieta española según el Î́ndice de alimentación saludable. Nutricion Hospitalaria, 26 (2), 330-336.

Prada, G., Herrén, O., \& Ortíz, R. (2008). Patrón alimentario y acceso a los alimentos en familias desplazadas en el municipio de Girón, Santander, Colombia. Revista Panamericana de Salud Publica/Pan American Journal of Public Health, 23(4), 257-263.

Ramos-Peña, E. G., Valdés, C., Cantú, P., Salinas, G., González, L., \& Berrún, L. (2007). Índice de marginación y el patrón de consumo alimentario familiar de Nuevo León. Papeles de Poblacion, 13(54), 265-285.

Ramos Peña, E. G. (2009). Consumo Alimentario en relación a determinantes socio-demográficos y una propuesta de política alimentaria en Nuevo León (Primera ed). Monterrey, México: Universidad Autónoma de Nuevo León.

SEDESOL. (2014). Diagnstico y Propuesta de Atención del Programa de Comedores Comunitarios. Consejo Nacional de Evaluación de la Política de Desarrollo Social. Recuperado el 9 de marzo de 2016 de https://www.gob.mx/cms/uploads/attachment/file/320 18/Diagnostico_2014_PCC_1_.pdf

Serra, L., Aranceta, J., \& Mataix, F. J. (2006). Nutrición y Salud Pública: métodos, bases científicas y aplicaciones (2da ed.). Barcelona, España: Elsevier Masson.

Sociedad Española de Nutrición Comunitaria. (2015). Piramide de la Alimentación saludable. Recuperado el 10 de febrero de 2016 de: http://www.nutricioncomunitaria.org/es/noticia/pirami de-de-la-alimentacion-saludable-senc-2015. 